Review Article

\title{
Antioxidative Potentials of Incretin-Based Medications: A Review of Molecular Mechanisms
}

\author{
Habib Yaribeygi ${ }^{D},{ }^{1}$ Mina Maleki, ${ }^{2}$ Thozhukat Sathyapalan, ${ }^{3}$ Tannaz Jamialahmadi, ${ }^{4,5}$ \\ and Amirhossein Sahebkar iD $6,7,8$ \\ ${ }^{1}$ Research Center of Physiology, Semnan University of Medical Sciences, Semnan, Iran \\ ${ }^{2}$ Chronic Kidney Disease Research Center, Shahid Beheshti University of Medical Sciences, Tehran, Iran \\ ${ }^{3}$ Academic Diabetes, Endocrinology and Metabolism, Hull York Medical School, University of Hull, UK \\ ${ }^{4}$ Department of Food Science and Technology, Quchan Branch, Islamic Azad University, Quchan, Iran \\ ${ }^{5}$ Department of Nutrition, Faculty of Medicine, Mashhad University of Medical Sciences, Mashhad, Iran \\ ${ }^{6}$ Biotechnology Research Center, Pharmaceutical Technology Institute, Mashhad University of Medical Sciences, Mashhad, Iran \\ ${ }^{7}$ Applied Biomedical Research Center, Mashhad University of Medical Sciences, Mashhad, Iran \\ ${ }^{8}$ School of Pharmacy, Mashhad University of Medical Sciences, Mashhad, Iran
}

Correspondence should be addressed to Habib Yaribeygi; habib.yari@yahoo.com

and Amirhossein Sahebkar; amir_saheb2000@yahoo.com

Received 4 March 2021; Revised 11 April 2021; Accepted 16 April 2021; Published 28 April 2021

Academic Editor: Marina Sokovi

Copyright @ 2021 Habib Yaribeygi et al. This is an open access article distributed under the Creative Commons Attribution License, which permits unrestricted use, distribution, and reproduction in any medium, provided the original work is properly cited.

Glucagon-like peptide 1 receptor agonists and dipeptidyl-peptidase 4 inhibitors are medications used for managing diabetes, mimicking the metabolic effects of incretin hormones. Recent evidence suggests that these medications have antioxidative potentials in the diabetic milieu. The pathophysiology of most diabetic complications involves oxidative stress. Therefore, if incretin-based antidiabetic medications can alleviate the free radicals involved in oxidative stress, they can potentially provide further therapeutic effects against diabetic complications. However, the molecular mechanisms by which these medications protect against oxidative stress are not fully understood. In the current review, we discuss the potential molecular mechanisms behind these pharmacologic agents' antioxidative properties.

\section{Introduction}

The incidence of diabetes mellitus (DM) is increasing in epidemic proportions globally [1]. DM carries considerable morbidity and takes up a significant proportion of health care burden and costs $[2,3]$. DM gives rise to various microvascular and macrovascular complications [4]. The development of diabetic complications involves different pathophysiologic pathways such as polyol pathway, hexosamine pathway, inflammatory responses, oxidative pathways, peroxidation, glucotoxicity, and lipotoxicity $[4,5]$. Although the exact pathophysiology of various diabetic complications is not clearly understood, there is growing evidence that oxidative stress plays a crucial part [6]. Hence, many antioxidative agents have been tried for treating DM and its complications
[7]. Glucagon-like peptide receptor-1 agonists (GLP-1RA) and dipeptidyl peptidase-4 inhibitors (DPP-4i) are classes of antidiabetic medications that are used in the management of DM mimicking the action of incretin hormones $[8,9]$. They reduce hyperglycemia through several mechanisms $[8,10]$.

In addition to their antihyperglycemic effects, recent evidence suggests that these medications could provide antioxidative effects $[11,12]$. If they can ameliorate DM-induced oxidative stress involved in the pathophysiology of diabetic complications, they could offer potential additional therapeutic effects in the management of diabetic complications [12-14]. However, the exact molecular mechanism by which these antidiabetic medications reduce oxidative stress is not fully elucidated. This review discusses the antioxidative properties of the incretin-based antihyperglycemic agents and the 
potential molecular mechanisms behind these effects. We conducted an extensive literature search using keywords of oxidative stress, diabetes mellitus, glucagon-like peptides, GLP-1 receptor agonist, and DPP-4 Inhibitor in databases such as PubMed, Scopus, the Web of Science, and Google Scholar between 2002 and 2020. However, the main limitation was the lack of enough related evidence. Still, we selected appropriate literature by extensively reading the available evidence and finding the most appropriate one demonstrating the possible involved molecular pathways.

\section{Classifications of Diabetes Mellitus}

DM is typically categorized into type 1 and type 2 diabetes [15]. Type $1 \mathrm{DM}$ (T1DM) is mainly referred to as lower circulatory insulin due to beta cell failure [15]. Type 2 DM (T2DM), which is the most prevalent form of DM, is mostly related to insulin resistance in insulin-dependent peripheral tissues [15]. Gestational diabetes is another form of DM which occurs in pregnant women likely due to hormonal variations during pregnancy [16]. In addition, there are other types of DM such as LADA (latent autoimmune diabetes in adults), maturity-onset diabetes of the young (MODY), secondary diabetes due to various pathological conditions such as pancreatitis, and secondary diabetes to certain medications, e.g., corticosteroids [17, 18]. As described below, GLP-1 mimetics exert antidiabetic effects in both type 1 and type $2 \mathrm{DM}[10]$.

\section{GLP-1RA and DPP-4i}

GLP-1RA is a class of antidiabetic medications that provide antihyperglycemic effects by mimicking incretin hormones' effects through stimulation of GLP-1 receptors $[19,20]$. Incretin hormones are a group of intestinal metabolic peptides such as GLP-1 and GIP (gastric inhibitory peptide), which reduce hyperglycemia via several pathways, including inhibition of glucagon, stimulating insulin secretion, delaying the gastric emptying, appetite suppression, declining intestinal nutrients absorption, improvement of lipid metabolism, and promoting pancreatic $\beta$-cell efficiency $[19,21-$ 24]. These medications have a specific receptor known as the GLP-1 receptor, a member of G-protein coupled receptors, which are principally located in surfaces of pancreatic $\beta$-cells $[22,25]$. GLP-1R activation is generally followed by cAMP (cyclic adenosine monophosphate) production leading to cellular depolarization and insulin secretion from the pancreatic $\beta$-cells $[22,26]$. GLP-1 is commonly metabolized by a protease known as dipeptidyl peptidase-4 (DPP-4), and thereby, DPP-4 inhibitors (i) provide antihyperglycemic effects by increasing the active levels of GLP-1 [27, 28]. Hence, the DPP-4i has similar but less potent effects than GLP-1RA (Table 1) [27].

3.1. Oxidative Stress and Diabetic Complications. Free radicals are active molecules that have unpaired electron(s) in the outer layer of their orbitals, which enables them to bind with an unpaired electron(s) in other molecules as well as biological particles [29]. These active species are generated physiologically through various biologic events and have physiologic roles in specific cellular processes [29]. The components of the antioxidative system neutralize the excess amounts of free radicals [29]. When the production of free radicals is increased or when the antioxidative system is weakened due to conditions such as DM, a pathologic state of oxidative stress is developed. Oxidative stress develops when free radicals overcome the ability antioxidative system to neutralize excess free radicals $[29,30]$. These excess free radicals will interfere with various physiological states, including complications resulting from diabetes [30]. Hence, the prevention of oxidative stress and improving redox state towards physiologic balance is crucial for preventing the development and progression of various disease states, including DM and its complications [31-34].

In addition to the direct deleterious effects on biologic elements, oxidative stress is a potent upstream event for various molecular pathways such as apoptosis, necrosis, autophagy, synthesis/release of nitric oxide, renin-angiotensin system (RAS), inflammatory responses, and autonomic nervous system $[30,35]$. It has bidirectional interactions with these molecular pathways, including those pathways involved in the development of diabetic complications [6, 36]. Oxidative stress initiates a cascade of events, resulting in the dysfunction of various tissues [6]. For example, excess amount of free radicals upregulate many types of inflammatory mediators such as tumor necrosis factor-alpha (TNF- $\alpha$ ), transforming growth factor-beta (TGF- $\beta$ ), interleukins (ILs), monocyte chemoattractant protein-1 (MCP-1), matrix metalloproteinase (MMP), nuclear factor-kappa $b$ (nf- $k b$ ), E-selectin, chemokines, and different forms of adhesion molecules [37-39]. These procytokines are involved in the pathophysiology of the development of diabetic nephropathy $[6$, 40]. Also, oxidative stress stimulates the expression and activity of apoptotic mechanisms such as p53, Bax/Bcl2 ratio, and caspases, leading to a higher rate of cellular apoptotic death $[41,42]$. Moreover, oxidative stress has potent direct effects on various biologic molecules such as peptides, lipids, carbohydrates, and, more importantly, nucleic acids, thereby negatively modifying their physiologic structures and function $[43,44]$. Hence, oxidative stress has significant roles in developing and progressing various diabetes-induced complications, including nephropathy, retinopathy, neuropathies, and cardiovascular diseases [31, 36, 45, 46].

\section{Antioxidative Potentials of GLP-1RA and DPP-4i}

There is growing evidence suggesting incretin-based antidiabetic medications can normalize the redox state in an oxidative milieu [47-49]; however, all molecular mechanisms involved are not elucidated [48]. In the following sections, we will review these interconnected molecular pathways (Figure 1; Table 2).

\section{Direct Effects}

5.1. Antioxidant Defense System: Roles for Nrf2, Sirt-1, and Sirt-3. Antioxidant defense system (ADS) is an intrinsic 
TABLE 1: Two main classes of incretin-based antidiabetic medications.

\begin{tabular}{cccc}
\hline & Approved drugs & Mechanisms of action \\
\hline GLP-1ra & Exenatide (exendin-4), albiglutide, liraglutide, lixisenatide, & Ref. \\
semaglutide, dulaglutide & Mimic hypoglycemic influences of incretin hormones & [19, 20] \\
DPP-4i & Sitagliptin, saxagliptin, vildagliptin, linagliptin & Increase the active circulatory levels of GLP-1 & [27, 28] \\
\hline
\end{tabular}

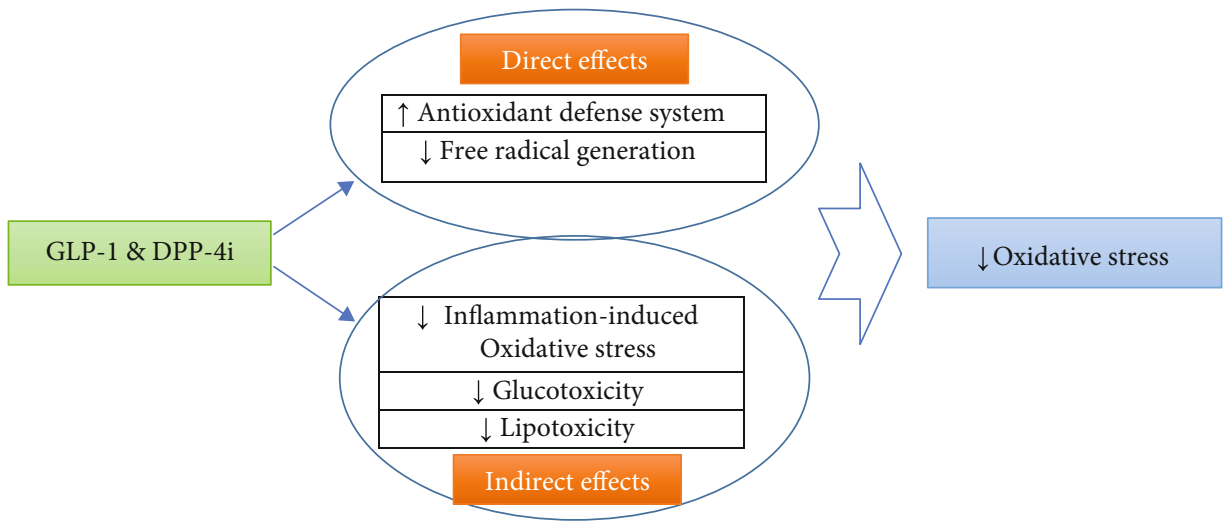

Figure 1: Possible antioxidant effects of GLP-1 receptor stimulation.

TABLE 2: Possible molecular pathways by which incretin-based antidiabetic medications protect against oxidative damages (SOD: superoxide dismutase; CAT: catalase; GPX: glutathione peroxidase; Nrf2: nuclear factor erythroid 2-related factor 2; AGEs: advanced glycation end products; Sirt: sirtuin; MDA: malondialdehyde).

\begin{tabular}{|c|c|c|c|}
\hline & Molecular mechanism & Effects on oxidative stress & Ref. \\
\hline \multirow{2}{*}{ Direct roles } & $\begin{array}{l}\text { Antioxidant defense } \\
\text { system }\end{array}$ & $\begin{array}{l}\text { Increase expression/activity of antioxidative elements such as SOD, } \\
\text { CAT, and GPX at least partly via Nrf2 and Sirt signaling pathways }\end{array}$ & {$[60,68,69,73]$} \\
\hline & Free radical generation & $\begin{array}{l}\text { Reduce the free radical generation thru several pathways such as suppressing } \\
\text { prooxidant enzymes and improving mitochondrial function }\end{array}$ & {$[54,60,86]$} \\
\hline \multirow{3}{*}{$\begin{array}{l}\text { Indirect } \\
\text { roles }\end{array}$} & $\begin{array}{l}\text { Inflammation-induced } \\
\text { oxidative stress }\end{array}$ & $\begin{array}{c}\text { Attenuate procytokines' expression/release leading to inflammation-induced } \\
\text { oxidative stress }\end{array}$ & {$[49,54]$} \\
\hline & Glucotoxicity & $\begin{array}{c}\text { Improve insulin signaling as well as glucose homeostasis leading to lower } \\
\text { amount of toxic byproduct as AGEs }\end{array}$ & {$[97,98]$} \\
\hline & Lipotoxicity & Reduce lipid metabolites such as MDA due to promoting lipid metabolism & {$[106-108]$} \\
\hline
\end{tabular}

molecular structure in most types of eukaryotic cells that neutralizes the different forms of free radical species, attenuates their harmful impacts, and protects against oxidative damages [50]. This protective system consists of both enzymatic and nonenzymatic elements such as superoxide dismutase (SOD), catalase (CAT), glutathione reductase (GR), and glutathione peroxidase (GPX), which are effective free radical scavengers and, thereby, their concentration, as well as activity, is a significant determinant of redox state in biologic milieu [51, 52]. Therefore, any agent that will be able to potentiate ADS will ameliorate various oxidative stresses and reduce the oxidative damages $[7,31,50]$.

There is growing evidence to suggest GLP-1 receptor induction potentiates the ADS through several pathways [53-55] [53-56]. Wang et al. in 2017 reported that after 24 hrs of GLP-1 therapy in human umbilical vein endothelial cells (HUVEC), cultured cells reversed the increase of high glucose-induced oxidative markers such as malondialdehyde
(MDA) and oxidized LDL (ox LDL) via upregulation of NQO1 (NAD(P)H Quinone Dehydrogenase 1), HO1 (Heme oxygenase 1), and GPX genes [53]. Induction of diabetes milieu in HUVECs is currently used in many studies [57]. In this design, HUVECs are cultured in a high glucose medium (i.e., $33 \mathrm{mM}$ glucose for about $72 \mathrm{~h}$ at $37^{\circ} \mathrm{C}$ ) to induce DM milieu [57]. Civantos et al. in 2017 found that sitagliptin attenuated DM-induced oxidative stress in renal tissues by the potentiation of ADS via miR-200a dependent pathway in diabetic rats [55]. Moreover, Shiraki and coworkers in 2012 observed that liraglutide increases the SOD-2, catalase, and GPX expression levels and thereby reduces TNF- $\alpha$ induced oxidative stress in the cultured HUVEC cell line [58]. Mangmool and coworkers in 2015 showed that exendin-4 upregulates ADS elements of CAT, GPX, and MnSOD in a dependent manner to Epac (exchange protein activated by cAMP) in cardiomyocytes of rats [59]. Bułdak and colleagues in 2015 reported that exenatide 
potentiated the antioxidative capacity of cultured human leukocytes increased expression of ADS elements [60]. Mohiuddin and coworkers in 2019 provided similar evidence in neuronal networks confirming GLP-1 receptor induction ameliorates oxidative damage in immortalized cultured dorsal root ganglions in the diabetic milieu [61]. This evidence demonstrates that GLP-1 receptor induction can potentiate ADS [54, 59-62].

Nuclear factor erythroid 2-related factor 2 (Nrf2) is a member of the basic leucine zipper (bZIP) nuclear transcription factors family, which enter the nucleus after phosphorylation, form heterodimers with other regulatory proteins, and bind to the specific regulatory regions of DNA known as ARE [63]. This factor is responsible for controlling antioxidant proteins' expression, thereby playing an essential role in keeping a normal redox state in cells [63]. Genetic knockdown of these transcription factors makes the tissues more vulnerable to oxidative injuries [64]. Also, pharmacologic activation of Nrf2 activity potentiates the ADS and protects tissues against oxidative damages [65-67].

We have evidence suggesting GLP-1 receptor induction stimulates the Nrf2 signaling pathways in various tissues [11, 68]. For example, Deng and coworkers in 2018 found that liraglutide induces Nrf2 signaling pathways and increased the expression level and activity of Nrf2 protein in the brain of diabetic rats [68]. Also, Fernández-Millán et al. in 2016 observed that GLP-1 increased the Nrf2 expression and activity in pancreatic islets [69]. Moreover, Kim and colleagues in 2017 established that exendin- 4 activates the Nrf2 signaling pathway and potentiates ADS element in rat insulinoma cells [70]. This evidence strongly suggests that GLP-1 receptor induction can stimulate Nrf2 signaling leading to an improved redox state [68-70].

Sirtuin (sirt) is a family of highly conserved proteins in mammals with at least seven members as Sirt1-7 [71]. Although these proteins' exact functions are not understood, some members of this family are involved in cell survival, life span, and response to various stimuli such as oxidative stress $[71,72]$. Recent evidence demonstrated that Sirt- 1 and Sirt-3 play a significant role in diabetes-related disorders and oxidative stress $[71,73]$. Sirt1 is a NAD- (nicotine-amide adenine)$^{+}$dependent deacetylase that controls oxidative stress responses and apoptosis via a p53 dependent mechanism, and recent studies suggest that its activity is decreased in DM [74, 75]. Sirt3 is another member of the sirtuin family, which is also involved in the oxidative response, especially in mitochondria, and controls metabolic pathways via $\mathrm{NAD}^{+}$-dependent deacetylase $[76,77]$.

There is evidence to suggest that incretin-based antidiabetic medications have interactions with sirt proteins [73, 78, 79]. GLP-1 receptor activation may increase or decline Sirt-dependent pathways in various conditions [80]. Zheng and coworkers in 2017 found that exenatide improved endoplasmic reticulum (ER) stress via sirt1 dependent pathway in hepatic cells of C57BL/6J mice [81]. Lee et al. in 2012 reported that exendin-4 upregulates sirt1 in hepatic cells of high-fat diet-dependent obese mice [82]. These findings suggest that GLP-1RA and DPP-4i can provide some antioxidative effects via sirt proteins. For example, Zeng and coworkers in 2016 demonstrated that exendin- 4 improved oxidative stress by promoting ADS potency via Sirt 1 and sirt 3 upregulation in the retina of diabetic rats [73]. The other possible molecular pathways, such as cAMP/PKA/ERK, have also been suggested by which GLP-1 upregulates the ADS elements [69]. It has been suggested that the Nrf2 signaling pathway can be also activated via the cAMP/PKA/ERK pathway [69]. Fernández-Millán and coworkers in 2016 found that GLP-1 increases antioxidant capacity and prevents oxidative damages via inducing Nrf2 protein expression and its translocation in a cAMP/PKA/ERK-dependent manner in diabetic beta cells [69]. So, GLP-1 mimetics are able to potentiate the activity of ADS elements via several molecular mechanisms.

5.2. Free Radical Generation. GLP-1 mimetic can reduce free radical generation through several pathways (Figure 2):

5.2.1. Prooxidant Enzymes. GLP-1 receptor activation has inhibitory effects on prooxidant enzymes and reduces their expression and activity [54]. Shiraki and colleagues in 2012 demonstrated that liraglutide downregulated the $\mathrm{gp} 91^{\text {phox }}$ and $\mathrm{p} 22^{\text {phox }}$ subunits of NADPH (nicotinamide adenine dinucleotide phosphate oxidase) oxidase (Nox) in cultured HUVECs [54]. This effect was accompanied by lower free radical generation and improvement in oxidative stress [54]. Similarly, Bułdak and coworkers in 2015 found that exenatide reduces free radical species via downregulation of $\mathrm{NADPH}$ oxidase in cultured human leukocytes [60]. Moreover, $\mathrm{Li}$ and colleagues in 2017 reported that pretreatment of HUVEC cells with GLP-1 reduces the $\mathrm{p} 47^{\text {phox }}$ subunit of $\mathrm{NADPH}$ oxidase- 4 and reduces DM-induced free radical generation [83]. Choi and colleagues in 2017 provided further evidence indicating gemigliptin (DPP-4i) and reduced mRNA expression of $\mathrm{p} 22^{\text {phox }}$ subunit of Nox, leading to lower free radical generation in rat model of adenineinduced chronic kidney disease [84].

5.2.2. Mitochondrial Dysfunction. Mitochondrial dysfunction is commonly associated with higher amounts of free radical production and oxidative stress [85]. There is some evidence to imply that GLP-1 receptor induction may improve mitochondrial function [80, 86]. Kang et al. in 2015 demonstrated that GLP-1 stimulates mitochondrial biogenesis, increases the mitochondrial membrane potential, and improves mitochondrial function in INS-1 (rat insulinoma cells) [86]. Góralska and coworkers in 2017 found that exendin-4 markedly improved mitochondrial efficiency in human adipocytes [80]. Also, Zhang and coworkers in 2017 demonstrated that alogliptin (DPP-4i) promoted mitochondrial function and attenuated mitochondrial free radical production in atrial tissues of diabetic rabbits [87].

5.2.3. Other Pathways. GLP-1RA and DPP-4i may reduce free radical generation through other molecular pathways [88, 89]. Laviola et al. in 2012 has reported that GLP-1 decreased free radical species by a JNK- (c-Jun N-terminal protein kinase-) dependent pathway and avoiding JNK phosphorylation in cultured human cardiac progenitor cells [89]. Mukai and coworkers in 2011 provided evidence indicating 


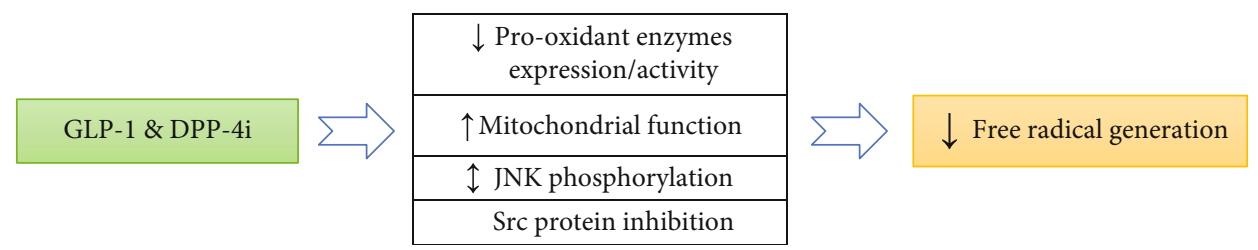

FIGURE 2: GLP-1 receptor induction reduces free radical species through four main molecular pathways (JNK: Janus kinase; Src: an adaptor protein).

TABLE 3: Clinical evidence about the antioxidative potentials of GLP-1RA and DPP-4i.

\begin{tabular}{lccc}
\hline Treatment & Population of study & Effects & Ref. \\
\hline Exenatide & 69 patients with T2DM & Reduced oxidative markers as MDA and oxLDL & [110] \\
GLP-1 & 15 patients with T1DM & Restored oxidative stress in endothelial cells, improved endothelial function & [12] \\
Liraglutide & 64 patients with T2DM & Provided cardioprotective effects via attenuating oxidative stress & [111] \\
GLP-1 & 60 patients with T2DM & Improved the palmitate-induced oxidative damages in cardiomyocytes & [113] \\
Liraglutide & 20 patients with T2DM & Declined oxidative markers and free radical generation & [112] \\
\hline
\end{tabular}

exendin- 4 decreases endogenous free radical species by inhibition of Src protein in islets of diabetic rats [88].

\section{Indirect Effects}

6.1. Inflammation-Induced Oxidative Stress. There is growing evidence that inflammatory responses can induce oxidative stress $[49,90]$. Inflammation-dependent oxidative damage is a known event in the inflamed tissues [90-92]. GLP-1R induction may ameliorate these phenomena [54, 93]. Shiraki and colleagues in 2012 stated that liraglutide suppresses TNF- $\alpha$ induced oxidative stress in cultured HUVEC cells [54]. They found that treatment with liraglutide markedly reduced inflammatory cytokines, which, in turn, attenuates inflammation-induced oxidative markers as MDA and oxLDL [54]. Alam et al. in 2015 reported that sitagliptin inhibited inflammation-induced oxidative damages in renal tissues of rats [49].

6.2. Glucotoxicity. Glucotoxicity refers to the toxic effects of hyperglycemia $[94,95]$. It can induce and exacerbate oxidative stress in several ways, including increasing the free radical species such as AGEs (advanced glycation end-products) and weakening the potency of ADS potency. An improvement in glucose homeostasis translates to a balanced redox state $[94,96]$. GLP-1 mimetics can improve glucose homeostasis and attenuate glucotoxicity by amplifying insulin signal transduction [97-99]. We have shown before that these agents can improve the insulin signaling pathway [10] through several mechanisms such as regulation of glucose transporters in 3T3-L1 adipocytes [100], upregulation of phosphorylated IR- $\beta$, IRS-1, Akt, and GSK-3beta (Glycogen synthase kinase 3 beta) in adipocytes [101], promoting Akt phosphorylation and cyclins A, D1, and E protein expression in adipocytes [102], and reducing toxic byproducts of AGEs leading to better glucose homeostasis in diabetic milieu $[103,104]$. Beyond the pathways mentioned above, they can indirectly normalize the redox state by promoting glucose metabolism $[97,98]$.
6.3. Lipotoxicity. Lipotoxicity occurs in patients with diabetes, mainly referring to altered lipid metabolism leading to a higher production of toxic byproducts such as MDA, which is the primary marker of oxidative damage [105]. GLP-1 receptor activation can improve lipid metabolism and reduction of toxic metabolites [106-108]. They can exert these metabolic effects in several ways, such as peroxisome proliferator-activated receptor alpha (PPAR- $\alpha$ ) [109]. This evidence suggests that incretin-based antidiabetic medications can reduce oxidative stress by improving lipid metabolism $[108,109]$.

\section{Clinical Evidence}

There is some clinical evidence to show that these medications protect against oxidative damages [12, 110] (Table 3). Bunck and coworkers in 2010 demonstrated that GLP-1 reduces oxidative markers such as MDA and oxLDL in patients with T2DM [110]. Also, Ceriello and coworkers in 2013 reported that GLP-1, through its antioxidative potentials, protects against endothelial dysfunction in patients with T1DM [12]. Moreover, Okada and colleagues in 2014 conducted a clinical trial on patients with T2DM, demonstrating that liraglutide provides cardioprotective effects via its antioxidative potentials [111]. Furthermore, Rizzo and coworkers in 2015 found that liraglutide attenuated oxidative stress markers in patients with T2DM [112] (Table 3).

\section{Conclusion}

GLP-1 mimetics improve oxidative stress through various direct and indirect pathways (Figure 1, Table 2). Direct antioxidative effects of incretin-based drugs are through their impact on ADS and free radical generation. They can potentiate the ADS by increasing the expression and activity of its components. They can also reduce free radical species through several pathways, such as prooxidant enzymes and mitochondrial function. These medications can also attenuate oxidative stress via indirect mechanisms such as lowering 
glucotoxicity, lipotoxicity, and inflammation-dependent oxidative stress. However, the limitations include a lack of enough direct experimental and clinical evidence confirming our suggested pathways, and, thereby, there is a need for more studies to confirm these effects. Especially, more clinical evidence is required to validate the findings of related experimental studies. Besides, potentially more molecular pathways involved in these effects may be discovered in the near future.

\section{Data Availability}

Not applicable.

\section{Conflicts of Interest}

The authors declare to have no conflict of interest in this study.

\section{Acknowledgments}

The authors are thankful to the "Research center of physiology, Semnan University of medical sciences (Semnan, Iran)" for providing technical supports.

\section{References}

[1] T. M. Abraham, K. M. Pencina, M. J. Pencina, and C. S. Fox, "Trends in diabetes incidence: the Framingham Heart Study," Diabetes Care, vol. 38, no. 3, pp. 482-487, 2015.

[2] J. da Rocha Fernandes, K. Ogurtsova, U. Linnenkamp et al., "IDF Diabetes Atlas estimates of 2014 global health expenditures on diabetes," Diabetes Research and Clinical Practice, vol. 117, pp. 48-54, 2016.

[3] E. J. Mayer-Davis, J. M. Lawrence, D. Dabelea et al., "Incidence trends of type 1 and type 2 diabetes among youths, 2002-2012," New England Journal of Medicine, vol. 376, no. 15, pp. 1419-1429, 2017.

[4] E. Bonora and R. A. DeFronzo, Diabetes complications, comorbidities and related disorders, Springer, 2018.

[5] I. B. Hirsch, "Glycemic variability and diabetes complications: does it matter? Of course it does!," Diabetes Care, vol. 38 , no. 8, pp. 1610-1614, 2015.

[6] F. Giacco and M. Brownlee, "Oxidative stress and diabetic complications," Circulation Research, vol. 107, no. 9, pp. 1058-1070, 2010.

[7] H. Yaribeygi, A. E. Butler, G. E. Barreto, and A. Sahebkar, "Antioxidative potential of antidiabetic agents: a possible protective mechanism against vascular complications in diabetic patients," Journal of Cellular Physiology, vol. 234, no. 3, pp. 2436-2446, 2019.

[8] R. A. DeFronzo, "Combination therapy with GLP-1 receptor agonist and SGLT2 inhibitor," Diabetes, Obesity and Metabolism, vol. 19, no. 10, pp. 1353-1362, 2017.

[9] M. Ashrafizadeh, H. Yaribeygi, S. L. Atkin, and A. Sahebkar, "Effects of newly introduced antidiabetic drugs on autophagy," Diabetes \& Metabolic Syndrome: Clinical Research \& Reviews, vol. 13, no. 4, pp. 2445-2449, 2019.

[10] H. Yaribeygi, T. Sathyapalan, and A. Sahebkar, "Molecular mechanisms by which GLP-1 RA and DPP-4i induce insulin sensitivity," Life Sciences, vol. 234, p. 116776, 2019.
[11] Y. Oh and H.-S. Jun, "Effects of glucagon-like peptide-1 on oxidative stress and Nrf2 signaling," International Journal of Molecular Sciences, vol. 19, no. 1, p. 26, 2018.

[12] A. Ceriello, A. Novials, E. Ortega et al., "Glucagon-like peptide 1 reduces endothelial dysfunction, inflammation, and oxidative stress induced by both hyperglycemia and hypoglycemia in type 1 diabetes," Diabetes Care, vol. 36, no. 8, pp. 2346-2350, 2013.

[13] A. Monji, T. Mitsui, Y. K. Bando, M. Aoyama, T. Shigeta, and T. Murohara, "Glucagon-like peptide-1 receptor activation reverses cardiac remodeling via normalizing cardiac steatosis and oxidative stress in type 2 diabetes," American Journal of Physiology-Heart and Circulatory Physiology, vol. 305, no. 3, pp. H295-H304, 2013.

[14] H. Yaribeygi, M. Maleki, T. Sathyapalan, T. Jamialahmadi, and A. Sahebkar, "Incretin-based therapies and reninangiotensin system: looking for new therapeutic potentials in the diabetic milieu," Life Sciences, vol. 256, p. 117916, 2020.

[15] Association, A.D., "2. Classification and diagnosis of diabetes," Diabetes care, vol. 40, Supplement 1, pp. S11-S24, 2017.

[16] J. de Faria Maraschin, Classification of diabetes, in Diabetes, Springer, 2013.

[17] K. S. O’Neal, J. L. Johnson, and R. L. Panak, "Recognizing and appropriately treating latent autoimmune diabetes in adults," Diabetes Spectrum, vol. 29, no. 4, pp. 249-252, 2016.

[18] Association, A.D., "Diagnosis and classification of diabetes mellitus," Diabetes care, vol. 37, Supplement_1, pp. S81S90, 2014.

[19] D. J. Drucker and M. A. Nauck, "The incretin system: glucagon-like peptide-1 receptor agonists and dipeptidyl peptidase- 4 inhibitors in type 2 diabetes," The Lancet, vol. 368 , no. 9548, pp. 1696-1705, 2006.

[20] M. Islam, "Insulinotropic effect of herbal drugs for management of diabetes mellitus: a congregational approach," Biosensors Journal, vol. 5, no. 142, p. 2, 2016.

[21] J. J. Meier, "GLP-1 receptor agonists for individualized treatment of type 2 diabetes mellitus," Nature Reviews Endocrinology, vol. 8, no. 12, pp. 728-742, 2012.

[22] L. L. Baggio and D. J. Drucker, "Biology of incretins: GLP-1 and GIP," Gastroenterology, vol. 132, no. 6, pp. 2131-2157, 2007.

[23] K. A. Scott and T. H. Moran, "The GLP-1 agonist exendin-4 reduces food intake in nonhuman primates through changes in meal size," American Journal of Physiology-Regulatory, Integrative and Comparative Physiology, vol. 293, no. 3, pp. R983-R987, 2007.

[24] X. Ding, N. K. Saxena, S. Lin, N. Gupta, and F. A. Anania, "Exendin-4, a glucagon-like protein-1 (GLP-1) receptor agonist, reverses hepatic steatosis in ob/ob mice," Hepatology, vol. 43, no. 1, pp. 173-181, 2006.

[25] H. Yaribeygi, A. Rashidy-Pour, S. L. Atkin, T. Jamialahmadi, and A. Sahebkar, "GLP-1 mimetics and cognition," Life Sciences, p. 118645, 2020.

[26] D. Wootten, J. Simms, C. Koole et al., "Modulation of the glucagon-like peptide-1 receptor signaling by naturally occurring and synthetic flavonoids," Journal of Pharmacology and Experimental Therapeutics, vol. 336, no. 2, pp. 540-550, 2011.

[27] Association, A.D., "2. Classification and diagnosis of diabetes: standards of medical care in diabetes-2018," Diabetes Care, vol. 41, Supplement 1, pp. S13-S27, 2018. 
[28] B. Ahren, "DPP-4 inhibitors," Best Practice \& Research Clinical Endocrinology \& Metabolism, vol. 21, no. 4, pp. 517-533, 2007.

[29] B. Halliwell and J. M. Gutteridge, Free radicals in biology and medicine, Oxford University Press, USA, 2015.

[30] W. Pryor, Free radicals in biology, vol. 6, Elsevier, 2012.

[31] H. Yaribeygi, M. T. Mohammadi, and A. Sahebkar, "Crocin potentiates antioxidant defense system and improves oxidative damage in liver tissue in diabetic rats," Biomedicine \& Pharmacotherapy, vol. 98, pp. 333-337, 2018.

[32] H. Yaribeygi, M. T. Mohammadi, R. Rezaee, and A. Sahebkar, "Fenofibrate improves renal function by amelioration of NOX-4, IL-18, and p53 expression in an experimental model of diabetic nephropathy," Journal of Cellular Biochemistry, vol. 119, no. 9, pp. 7458-7469, 2018.

[33] H. Yaribeygi and M. Mohammadi, "Protective effect of crocin on kidney performance in chronic uncontrolled hyperglycemia-induced nephropathy in rat," Journal of Advances in Medical and Biomedical Research, vol. 25, 2017.

[34] H. Yaribeygi, V. Zare, A. E. Butler, G. E. Barreto, and A. Sahebkar, "Antidiabetic potential of saffron and its active constituents," Journal of Cellular Physiology, vol. 234, no. 6, pp. 8610-8617, 2019.

[35] H. Yaribeygi, Y. Panahi, B. Javadi, and A. Sahebkar, "The underlying role of oxidative stress in neurodegeneration: a mechanistic review," CNS \& Neurological Disorders-Drug Targets (Formerly Current Drug Targets-CNS \& Neurological Disorders), vol. 17, no. 3, pp. 207-215, 2018.

[36] D. M. Niedowicz and D. L. Daleke, "The role of oxidative stress in diabetic complications," Cell Biochemistry and Biophysics, vol. 43, no. 2, pp. 289-330, 2005.

[37] J. F. Navarro-González, C. Mora-Fernández, M. M. de Fuentes, and J. García-Pérez, "Inflammatory molecules and pathways in the pathogenesis of diabetic nephropathy," Nature Reviews Nephrology, vol. 7, no. 6, pp. 327-340, 2011.

[38] J. Donate-Correa, E. Martín-Núñez, M. Muros-de-Fuentes, C. Mora-Fernández, and J. F. Navarro-González, "Inflammatory cytokines in diabetic nephropathy," Journal of Diabetes Research, vol. 2015, 9 pages, 2015.

[39] G. Garibotto, A. Carta, D. Picciotto, F. Viazzi, and D. Verzola, "Toll-like receptor-4 signaling mediates inflammation and tissue injury in diabetic nephropathy," Journal of Nephrology, vol. 30, no. 6, pp. 719-727, 2017.

[40] C. P. Domingueti, L. M. S.'. A. Dusse, M. . G. Carvalho, L. P. de Sousa, K. B. Gomes, and A. P. Fernandes, "Diabetes mellitus: the linkage between oxidative stress, inflammation, hypercoagulability and vascular complications," Journal of Diabetes and its Complications, vol. 30, no. 4, pp. 738-745, 2016.

[41] S. Sifuentes-Franco, D. E. Padilla-Tejeda, S. Carrillo-Ibarra, and A. G. Miranda-Díaz, "Oxidative stress, apoptosis, and mitochondrial function in diabetic nephropathy," International Journal of Endocrinology, vol. 2018, 13 pages, 2018.

[42] H. Yaribeygi, F. Lhaf, T. Sathyapalan, and A. Sahebkar, "Effects of novel antidiabetes agents on apoptotic processes in diabetes and malignancy: implications for lowering tissue damage," Life Sciences, vol. 231, p. 116538, 2019.

[43] G. Cenini, A. Lloret, and R. Cascella, "Oxidative stress in neurodegenerative diseases: from a mitochondrial point of view," Oxidative Medicine and Cellular Longevity, vol. 2019, 18 pages, 2019.
[44] A. Thapa and N. Carroll, "Dietary modulation of oxidative stress in Alzheimer's disease," International Journal of Molecular Sciences, vol. 18, no. 7, p. 1583, 2017.

[45] H. Yaribeygi, F. R. Farrokhi, R. Rezaee, and A. Sahebkar, "Oxidative stress induces renal failure: a review of possible molecular pathways," Journal of Cellular Biochemistry, vol. 119, no. 4, pp. 2990-2998, 2018.

[46] H. Yaribeygi, M. T. Mohammadi, R. Rezaee, and A. Sahebkar, "Crocin improves renal function by declining Nox-4, IL-18, and p53 expression levels in an experimental model of diabetic nephropathy," Journal of Cellular Biochemistry, vol. 119, no. 7, pp. 6080-6093, 2018.

[47] H. Hendarto, T. Inoguchi, Y. Maeda et al., "GLP-1 analog liraglutide protects against oxidative stress and albuminuria in streptozotocin-induced diabetic rats via protein kinase Amediated inhibition of renal NAD (P) H oxidases," Metabolism, vol. 61, no. 10, pp. 1422-1434, 2012.

[48] D. de Lima Ávila, G. R. de Araújo, M. Silva et al., "Vildagliptin ameliorates oxidative stress and pancreatic beta cell destruction in type 1 diabetic rats," Archives of Medical Research, vol. 44, no. 3, pp. 194-202, 2013.

[49] M. A. Alam, M. R. H. Chowdhury, P. Jain, M. A. T. Sagor, and H. M. Reza, "DPP-4 inhibitor sitagliptin prevents inflammation and oxidative stress of heart and kidney in two kidney and one clip (2K1C) rats," Diabetology \& Metabolic Syndrome, vol. 7, no. 1, 2015.

[50] M. Kobayashi, L. Li, N. Iwamoto et al., "The antioxidant defense system Keap1-Nrf2 comprises a multiple sensing mechanism for responding to a wide range of chemical compounds," Molecular and Cellular Biology, vol. 29, no. 2, pp. 493-502, 2009.

[51] S. I. Zandalinas, D. Balfagón, V. Arbona, and A. GómezCadenas, "Modulation of antioxidant defense system is associated with combined drought and heat stress tolerance in citrus," Frontiers in Plant Science, vol. 8, p. 953, 2017.

[52] C. Rico, J. Peralta-Videa, and J. Gardea-Torresdey, "Chemistry, biochemistry of nanoparticles, and their role in antioxidant defense system in plants," in Nanotechnology and Plant Sciences, pp. 1-17, Springer, 2015.

[53] L. Guo, Y. Qiao, L. Zhang, and Q. Pan, "Protective effect and molecular mechanism of GLP-1 on the high glucose-induced endothelial cell injury," Journal of Hainan Medical University, vol. 23, no. 21, pp. 1-4, 2017.

[54] A. Shiraki, J. I. Oyama, H. Komoda, and K. Node, "Glucagonlike peptide 1 analog liraglutide reduces TNF- $\alpha$-induced oxidative stress and inflammation," Journal of the American College of Cardiology, vol. 59, no. 13, p. E904, 2012.

[55] E. Civantos, E. Bosch, E. Ramirez et al., "Sitagliptin ameliorates oxidative stress in experimental diabetic nephropathy by diminishing the miR-200a/Keap-1/Nrf2 antioxidant pathway," Diabetes, metabolic syndrome and obesity: targets and therapy, vol. Volume 10, pp. 207-222, 2017.

[56] Y. L. Wu, J. Huang, J. Liu et al., "Protective effect of recombinant human glucagon-like peptide-1 (rhGLP-1) pretreatment in STZ-induced diabetic mice," Journal of Peptide Science, vol. 17, no. 7, pp. 499-504, 2011.

[57] J. Zhang, Y. Guo, W. Ge, X. Zhou, and M. Pan, "High glucose induces apoptosis of HUVECs in a mitochondria-dependent manner by suppressing hexokinase 2 expression," Experimental and Therapeutic Medicine, vol. 18, no. 1, pp. 621629, 2019. 
[58] A. Shiraki, J. I. Oyama, H. Komoda et al., "The glucagon-like peptide 1 analog liraglutide reduces TNF- $\alpha$-induced oxidative stress and inflammation in endothelial cells," Atherosclerosis, vol. 221, no. 2, pp. 375-382, 2012.

[59] S. Mangmool, P. Hemplueksa, W. Parichatikanond, and N. Chattipakorn, "Epac is required for GLP-1R-mediated inhibition of oxidative stress and apoptosis in cardiomyocytes," Molecular Endocrinology, vol. 29, no. 4, pp. 583-596, 2015.

[60] Ł. Bułdak, K. Łabuzek, R. J. Bułdak, G. Machnik, A. Bołdys, and B. Okopień, "Exenatide (a GLP-1 agonist) improves the antioxidative potential of in vitro cultured human monocytes/macrophages," Naunyn-Schmiedeberg's Archives of Pharmacology, vol. 388, no. 9, pp. 905-919, 2015.

[61] M. S. Mohiuddin, T. Himeno, R. Inoue et al., "Glucagon-like peptide-1 receptor agonist protects dorsal root ganglion neurons against oxidative insult," Journal of Diabetes Research, vol. 2019, 10 pages, 2019 .

[62] M. Lotfy, J. Singh, H. Rashed, S. Tariq, E. Zilahi, and E. Adeghate, "Mechanism of the beneficial and protective effects of exenatide in diabetic rats," The Journal of Endocrinology, vol. 220, no. 3, pp. 291-304, 2014.

[63] T. Nguyen, P. Nioi, and C. B. Pickett, "The Nrf2-antioxidant response element signaling pathway and its activation by oxidative stress," Journal of Biological Chemistry, vol. 284, no. 20, pp. 13291-13295, 2009.

[64] B. Yang, J. Fu, H. Zheng et al., "Deficiency in the nuclear factor E2-related factor 2 renders pancreatic $\beta$-cells vulnerable to arsenic-induced cell damage," Toxicology and Applied Pharmacology, vol. 264, no. 3, pp. 315-323, 2012.

[65] A. Kode, S. Rajendrasozhan, S. Caito, S. R. Yang, I. L. Megson, and I. Rahman, "Resveratrol induces glutathione synthesis by activation of Nrf2 and protects against cigarette smokemediated oxidative stress in human lung epithelial cells," American Journal of Physiology-Lung Cellular and Molecular Physiology, vol. 294, no. 3, pp. L478-L488, 2008.

[66] Q. Ma, "Role of nrf2 in oxidative stress and toxicity," Annual Review of Pharmacology and Toxicology, vol. 53, no. 1, pp. 401-426, 2013.

[67] S. Li, N. D. Vaziri, Y. Masuda et al., "Pharmacological activation of Nrf2 pathway improves pancreatic islet isolation and transplantation," Cell Transplantation, vol. 24, no. 11, pp. 2273-2283, 2015.

[68] C. Deng, J. Cao, J. Han et al., "Liraglutide activates the Nrf2/HO-1 antioxidant pathway and protects brain nerve cells against cerebral ischemia in diabetic rats," Computational Intelligence and Neuroscience, vol. 2018, 7 pages, 2018.

[69] E. Fernández-Millán, M. A. Martín, L. Goya et al., "Glucagonlike peptide-1 improves beta-cell antioxidant capacity via extracellular regulated kinases pathway and Nrf2 translocation," Free Radical Biology and Medicine, vol. 95, pp. 16-26, 2016.

[70] M.-H. Kim, E. H. Kim, H. S. Jung, D. Yang, E. Y. Park, and H. S. Jun, "EX4 stabilizes and activates $\mathrm{Nrf2}$ via $\mathrm{PKC} \delta$, contributing to the prevention of oxidative stress-induced pancreatic beta cell damage," Toxicology and Applied Pharmacology, vol. 315, pp. 60-69, 2017.

[71] Y. Kanfi, S. Naiman, G. Amir et al., "The sirtuin SIRT6 regulates lifespan in male mice," Nature, vol. 483, no. 7388, pp. 218-221, 2012.

[72] J. A. Baur, Z. Ungvari, R. K. Minor, D. G. le Couteur, and R. de Cabo, "Are sirtuins viable targets for improving health- span and lifespan?," Nature Reviews Drug Discovery, vol. 11, no. 6, pp. 443-461, 2012.

[73] Y. Zeng, K. Yang, F. Wang et al., "The glucagon like peptide 1 analogue, exendin-4, attenuates oxidative stress-induced retinal cell death in early diabetic rats through promoting Sirtl and Sirt3 expression," Experimental Eye Research, vol. 151, pp. 203-211, 2016.

[74] S. Kubota, Y. Ozawa, T. Kurihara et al., "Roles of AMPactivated protein kinase in diabetes-induced retinal inflammation," Investigative Ophthalmology \& Visual Science, vol. 52, no. 12, pp. 9142-9148, 2011.

[75] Z. Zheng, H. Chen, J. Li et al., "Sirtuin 1-mediated cellular metabolic memory of high glucose via the LKB1/AMPK/ROS pathway and therapeutic effects of metformin," Diabetes, vol. 61, no. 1, pp. 217-228, 2012.

[76] S. Someya, W. Yu, W. C. Hallows et al., "Sirt3 mediates reduction of oxidative damage and prevention of agerelated hearing loss under caloric restriction," Cell, vol. 143, no. 5, pp. 802-812, 2010.

[77] N. R. Sundaresan, M. Gupta, G. Kim, S. B. Rajamohan, A. Isbatan, and M. P. Gupta, "Sirt3 blocks the cardiac hypertrophic response by augmenting Foxo3a-dependent antioxidant defense mechanisms in mice," The Journal of Clinical Investigation, vol. 119, no. 9, pp. 2758-2771, 2009.

[78] P.-O. Bastien-Dionne, L. Valenti, N. Kon, W. Gu, and J. Buteau, "Glucagon-like peptide 1 inhibits the sirtuin deacetylase SirT1 to stimulate pancreatic $\beta$-cell mass expansion," Diabetes, vol. 60, no. 12, pp. 3217-3222, 2011.

[79] F. Xu, Z. Li, X. Zheng et al., "SIRT1 mediates the effect of GLP-1 receptor agonist exenatide on ameliorating hepatic steatosis," Diabetes, vol. 63, no. 11, pp. 3637-3646, 2014.

[80] J. Góralska, A. Śliwa, A. Gruca et al., "Glucagon-like peptide1 receptor agonist stimulates mitochondrial bioenergetics in human adipocytes," Acta Biochimica Polonica, vol. 64, no. 3, pp. 423-429, 2017, -429-423-429.

[81] X. Zheng, F. Xu, H. Liang et al., "SIRT1/HSF1/HSP pathway is essential for exenatide-alleviated, lipid-induced hepatic endoplasmic reticulum stress," Hepatology, vol. 66, no. 3, pp. 809-824, 2017.

[82] J. Lee, S. W. Hong, S. W. Chae et al., "Exendin-4 improves steatohepatitis by increasing Sirtl expression in high-fat diet-induced obese C57BL/6J mice," PLoS One, vol. 7, no. 2, article e31394, 2012.

[83] Q. Li, Y. Lin, S. Wang, L. Zhang, and L. Guo, "GLP-1 inhibits high-glucose-induced oxidative injury of vascular endothelial cells," Scientific Reports, vol. 7, no. 1, p. 8008, 2017.

[84] S.-Y. Choi, H. M. Ryu, E. J. Oh et al., "Dipeptidyl peptidase-4 inhibitor gemigliptin protects against vascular calcification in an experimental chronic kidney disease and vascular smooth muscle cells," PLoS One, vol. 12, no. 7, article e0180393, 2017.

[85] M. T. Lin and M. F. Beal, "Mitochondrial dysfunction and oxidative stress in neurodegenerative diseases," Nature, vol. 443 , no. 7113, pp. 787-795, 2006.

[86] M. Y. Kang, T. J. Oh, and Y. M. Cho, “Glucagon-like peptide1 increases mitochondrial biogenesis and function in INS-1 rat insulinoma cells," Endocrinology and Metabolism, vol. 30, no. 2, pp. 216-220, 2015.

[87] X. Zhang, Z. Zhang, Y. Zhao et al., "Alogliptin, a dipeptidyl peptidase-4 inhibitor, alleviates atrial remodeling and improves mitochondrial function and biogenesis in diabetic rabbits," Journal of the American Heart Association, vol. 6, no. 5, 2017. 
[88] E. Mukai, S. Fujimoto, H. Sato et al., "Exendin-4 suppresses SRC activation and reactive oxygen species production in diabetic Goto-Kakizaki rat islets in an Epac-dependent manner," Diabetes, vol. 60, no. 1, pp. 218-226, 2011.

[89] L. Laviola, A. Leonardini, M. Melchiorre et al., "Glucagonlike peptide-1 counteracts oxidative stress-dependent apoptosis of human cardiac progenitor cells by inhibiting the activation of the c-Jun $\mathrm{N}$-terminal protein kinase signaling pathway," Endocrinology, vol. 153, no. 12, pp. 5770-5781, 2012.

[90] K. Esposito, F. Nappo, R. Marfella et al., "Inflammatory cytokine concentrations are acutely increased by hyperglycemia in humans: role of oxidative stress," Circulation, vol. 106, no. 16, pp. 2067-2072, 2002.

[91] S. K. Biswas, "Does the interdependence between oxidative stress and inflammation explain the antioxidant paradox?," Oxidative Medicine and Cellular Longevity, vol. 2016, Article ID 5698931, 9 pages, 2016.

[92] H. Solleiro-Villavicencio and S. Rivas-Arancibia, "Effect of chronic oxidative stress on neuroinflammatory response mediated by CD4+ T cells in neurodegenerative diseases," Frontiers in Cellular Neuroscience, vol. 12, p. 114, 2018.

[93] H. Yaribeygi, M. Maleki, T. Sathyapalan, T. Jamialahmadi, and A. Sahebkar, "Anti-inflammatory potentials of incretinbased therapies used in the management of diabetes," Life Sciences, vol. 241, p. 117152, 2020.

[94] M. Oelze, S. Kröller-Schön, P. Welschof et al., "The sodiumglucose co-transporter 2 inhibitor empagliflozin improves diabetes-induced vascular dysfunction in the streptozotocin diabetes rat model by interfering with oxidative stress and glucotoxicity,” PLoS One, vol. 9, no. 11, article e112394, 2014.

[95] J. Wu and L.-J. Yan, "Streptozotocin-induced type 1 diabetes in rodents as a model for studying mitochondrial mechanisms of diabetic $\beta$ cell glucotoxicity," Diabetes, metabolic syndrome and obesity: targets and therapy, vol. 8, p. 181, 2015.

[96] Z. Asemi, M. Jamilian, E. Mesdaghinia, and A. Esmaillzadeh, "Effects of selenium supplementation on glucose homeostasis, inflammation, and oxidative stress in gestational diabetes: randomized, double-blind, placebo-controlled trial," Nutrition, vol. 31, no. 10, pp. 1235-1242, 2015.

[97] B. Ahrén and J. E. Foley, "Improved glucose regulation in type 2 diabetic patients with DPP-4 inhibitors: focus on alpha and beta cell function and lipid metabolism," Diabetologia, vol. 59, no. 5, pp. 907-917, 2016.

[98] W. Alsalim, O. Göransson, R. D. Carr et al., "Effect of singledose DPP-4 inhibitor sitagliptin on $\beta$-cell function and incretin hormone secretion after meal ingestion in healthy volunteers and drug-naïve, well-controlled type 2 diabetes subjects," Diabetes, Obesity and Metabolism, vol. 20, no. 4, pp. 1080-1085, 2018.

[99] P. Nadkarni, O. G. Chepurny, and G. G. Holz, "Regulation of glucose homeostasis by GLP-1," in Progress in molecular biology and translational science, pp. 23-65, Elsevier, 2014.

[100] Y. Wang, H. K. Kole, C. Montrose-Rafizadeh, R. Perfetti, M. Bernier, and J. M. Egan, "Regulation of glucose transporters and hexose uptake in 3T3-L1 adipocytes: glucagonlike peptide-1 and insulin interactions," Journal of Molecular Endocrinology, vol. 19, no. 3, pp. 241-248, 1997.

[101] H. Gao, X. Wang, Z. Zhang et al., "GLP-1 amplifies insulin signaling by up-regulation of IR $\beta$, IRS- 1 and Glut4 in 3T3L1 adipocytes," Endocrine, vol. 32, no. 1, pp. 90-95, 2007.
[102] D. Kawamori, J. Shirakawa, C. W. Liew et al., "GLP-1 signalling compensates for impaired insulin signalling in regulating beta cell proliferation in $\beta$ IRKO mice," Diabetologia, vol. 60, no. 8, pp. 1442-1453, 2017.

[103] J.-T. Chang, Y. J. Liang, C. Y. Hsu et al., "Glucagon-like peptide receptor agonists attenuate advanced glycation end products-induced inflammation in rat mesangial cells," BMC Pharmacology and Toxicology, vol. 18, no. 1, p. 67, 2017.

[104] S. Zhu, Y. Gu, W. Wang et al., "Sitagliptin ameliorates advanced glycation end-product (AGE)-induced degradation of extracellular matrix in human primary chondrocytes," American Journal of Translational Research, vol. 11, no. 5, pp. 2775-2783, 2019.

[105] R. A. Defronzo, "Dysfunctional fat cells, lipotoxicity and type 2 diabetes," International Journal of Clinical Practice, vol. 58, pp. 9-21, 2004.

[106] E. Abbasi Oshaghi, “Glucagon like peptide-1 (GLP-1) a novel therapeutic strategy in non-alcoholic fatty liver disease (NAFLD)," Avicenna Journal of Medical Biochemistry, vol. 5, no. 2, pp. 54-59, 2017.

[107] E. Bahne, M. Hansen, A. Brønden, D. P. Sonne, T. Vilsbøll, and F. K. Knop, "Involvement of glucagon-like peptide-1 in the glucose-lowering effect of metformin," Diabetes, Obesity and Metabolism, vol. 18, no. 10, pp. 955-961, 2016.

[108] M. J. Armstrong, D. Hull, K. Guo et al., "Glucagon-like peptide 1 decreases lipotoxicity in non-alcoholic steatohepatitis," Journal of Hepatology, vol. 64, no. 2, pp. 399-408, 2016.

[109] L. Wu, K. Wang, W. Wang et al., "Glucagon-like peptide-1 ameliorates cardiac lipotoxicity in diabetic cardiomyopathy via the PPAR $\alpha$ pathway," Aging Cell, vol. 17, no. 4, article e12763, 2018.

[110] M. C. Bunck, A. Cornér, B. Eliasson et al., "One-year treatment with exenatide vs. insulin glargine: effects on postprandial glycemia, lipid profiles, and oxidative stress," Atherosclerosis, vol. 212, no. 1, pp. 223-229, 2010.

[111] K. Okada, K. Kotani, H. Yagyu, A. Ando, J. I. Osuga, and S. Ishibashi, "Effects of treatment with liraglutide on oxidative stress and cardiac natriuretic peptide levels in patients with type 2 diabetes mellitus," Endocrine, vol. 47, no. 3, pp. 962-964, 2014.

[112] M. Rizzo, N. Abate, M. Chandalia et al., "Liraglutide reduces oxidative stress and restores heme oxygenase-1 and ghrelin levels in patients with type 2 diabetes: a prospective pilot study," The Journal of Clinical Endocrinology \& Metabolism, vol. 100, no. 2, pp. 603-606, 2015.

[113] S. Ravassa, J. Beaumont, A. Huerta et al., "Association of low GLP-1 with oxidative stress is related to cardiac disease and outcome in patients with type 2 diabetes mellitus: a pilot study," Free Radical Biology and Medicine, vol. 81, pp. 1-12, 2015. 